\title{
Desarrollo de un modelo virtual para el conformado de aceros inoxidables
}

\section{Development of a Virtual Model for Stainless Steel Forming}

\author{
Ing. Salvador Bravo Vargas \\ Centro de Tecnología Avanzada; Bombardier Transportation (CIATEQ, A.C.), Querétaro, México. \\ salvador.bravo@rail.bombardier.com
}

Recibido: 13 de junio 2017

Aceptado: 27 de setiembre 2017

\begin{abstract}
Resumen
En este trabajo se presenta un modelo virtual en ANSYS del proceso de conformado en aceros inoxidables que permite la obtención de la fibra neutra. La fibra neutra, también conocida como factor K, permite el cálculo del desarrollo de una pieza para su posterior conformado. Dada la complejidad de los procesos de conformado en la industria, como lo son el doblado, embutido y estampado, es necesario realizar el análisis computacional de los mismos. Es importante disponer de modelos que permitan conocer el comportamiento de los materiales durante su procesamiento para disminuir los posteriores errores de manufactura. Este trabajo propone un método de análisis por elementos finitos para el conformado de aceros inoxidables y presenta la comparativa con la experimentación física. Se ha caracterizado el proceso particular de doblado y se ha hecho la comparativa con probetas físicas de los aceros inoxidables 201 y 304, la cual muestra una diferencia de apenas el 0.4 por ciento entre ambas. El factor K obtenido puede ser utilizado directamente para cálculos analíticos para el desarrollo de piezas, o en los softwares de plegado de chapa metálica.
\end{abstract}

\section{Palabras clave:}

Conformado, fibra neutra, factor $\mathrm{K}$, aceros inoxidables, CAD, análisis por elementos finitos.

\begin{abstract}
In this paper, a virtual model in ANSYS for the forming process of stainless steels which allows obtaining the neutral fiber is presented. The neutral fiber, also known as $\mathrm{K}$ factor, allows determining the blank of a piece for its later forming. Due to the complexity of the forming processes in the industry, such as bending, shaping, and stamping, it is necessary to perform computational analyses. It is important to count on models that allow understanding the behavior of the material during its processing to reduce subsequent manufacturing mistakes. In this paper, a finite element-based analysis method for the forming process of stainless steels is proposed, and also its comparison with physical experimentation is presented. A particular bending process was characterized and its comparison with physical tests of 201 and 304 stainless steels was
\end{abstract}


undertaken. Such comparison shows a difference of 0.4 percent between them. The obtained K factor could be used directly for analytical calculations of the blank of parts or for the software development of sheet metal forming.

Keywords:

Forming process, neutral fiber, $\mathrm{K}$ factor, stainless steel, $\mathrm{CAD}$, finite element analysis. 


\section{INTRODUCCIÓN}

El conformado es un proceso de manufactura ampliamente utilizado en la industria metalmecánica debido a su flexibilidad, bajo costo y gran capacidad para procesar geometrías de elevada complejidad en láminas metálicas (Castro, 2010). Este proceso es aplicado en diversas industrias como la automovilística, la aeroespacial, la naval, la ferroviaria, la alimentaria, la de la construcción, entre otras (Amor, 2009). Para obtener una pieza conformada dentro de las tolerancias dimensionales del diseño, existen importantes variables que deben considerarse, tales como: el retroceso elástico o springback, las propiedades mecánicas del material y la ubicación de la fibra neutra o factor K (Kalpakjian y Schmid, 2006).

La ubicación correcta de la fibra neutra en el proceso de conformado permite que las piezas sean dobladas sin tener problemas de dimensiones, se eliminan tiempos de retrabajos y las piezas tendrán un comportamiento mecánico adecuado. La aportación de este trabajo consiste en ofrecer la ubicación correcta de la fibra neutra para los aceros inoxidables 301 y 304. La determinación de este parámetro busca eliminar el método de prueba y error empleado en la práctica industrial, disminuyendo el costo que este implica.

En este trabajo se propone un modelo virtual en ANSYS que puede ser utilizado en una gran cantidad de procesos de conformado, con la debida caracterización de cada uno de los materiales que se necesiten evaluar. Este modelo permite ubicar la fibra neutra en los materiales evaluados, dependiendo del espesor de los mismos. El modelo permite generar una tabla de valores de la posición de la fibra neutra que pueden ser utilizada para que el desarrollo de piezas se realice de forma precisa garantizando que los diseños conformados cumplan con las especificaciones dadas.

El documento está organizado de la siguiente manera: la Sección II muestra los antecedentes de métodos analíticos y técnicas de simulación empleados en el proceso de conformado; la Sección III presenta el análisis físico del conformado en probetas; la Sección IV presenta la caracterización y análisis por medio del método de elementos finitos; la Sección V muestra los resultados de los análisis; y por último, la Sección VI expone las conclusiones del trabajo.

\section{ANTECEDENTES}

Durante la década de 1980 se comenzó a estudiar con mayor profundidad el conformado del acero mediante el uso de herramientas analíticas, como es el caso del método de elementos finitos (Banabic, 2010). Dichos estudios se debieron, en gran parte, a la necesidad de la industria automotriz de reducir los costos ocasionados por los métodos de prueba y error en sus procesos de conformado. A inicios de la década de 1990 se establecieron algunas técnicas que simulaban el uso del par de dados empleados en el conformado y el material que sería procesado (García-Romeu, 2005). En 1992 se perfeccionaron métodos como los de Lange (1992) y Marciniak, Duncan y Hu (2002), que muestran la aplicación directa de la ingeniería de la plasticidad a los procesos industriales y su simulación tridimensional. Del mismo modo, se amplió el uso de las simulaciones, haciéndolo extensivo al estampado, embutido profundo, punzonado y doblado de tubería (Webb y Hardt, 1991). 
El uso de software que incluye análisis por elementos finitos, como ANSYS (Moaveni, 1999), permite el estudio de este tipo de procesos, que incluyen las variables mecánicas de los materiales y las complejas geometrías de las herramientas, y convierten las pruebas físicas en algo que demanda demasiado tiempo y costos elevados. Existe un factor de vital importancia dentro del conformado que se conoce como fibra neutra o factor $\mathrm{K}$, el cual se debe estimar con el fin de que la pieza cumpla con las medidas que están especificadas en los diseños. Actualmente, en la industria se manejan tablas elaboradas por la ASM (1988) que estiman el factor K de diferentes aceros; sin embargo, estas reflejan únicamente el conocimiento empírico de los talleres de conformado y no son obtenidas por un método analítico. Por tanto, en la práctica presentan resultados poco precisos que provocan que las piezas conformadas estén fuera de las especificaciones de diseño, generando costos extras. Es necesaria la realización de un estudio que estime la ubicación de la fibra neutra para conocer el desarrollo o tamaño al cuál se deberá cortar la pieza desplegada, para su posterior conformado. Actualmente la mayoría de las investigaciones recientes se enfocan en las secuencias propias del conformado con el fin de reducir tiempos de manufactura (Zahid, Waheed y Liaqat, 2016) y también sobre el conocimiento de la variación del retroceso elástico, conocido como springback en los diversos procesos de conformado (Balon, Swiatoniowski y Szostak, 2016). Hasta el día de hoy no existe algún estudio sobre la ubicación del factor K en los aceros inoxidables.

En este trabajo se propone un modelo virtual para determinar la ubicación de la fibra neutra, para que una pieza desplegada, como la mostrada en la figura 1, sea conformada dentro de las especificaciones de diseño por parte del área de ingeniería del producto, como la presentada en la figura 2 .

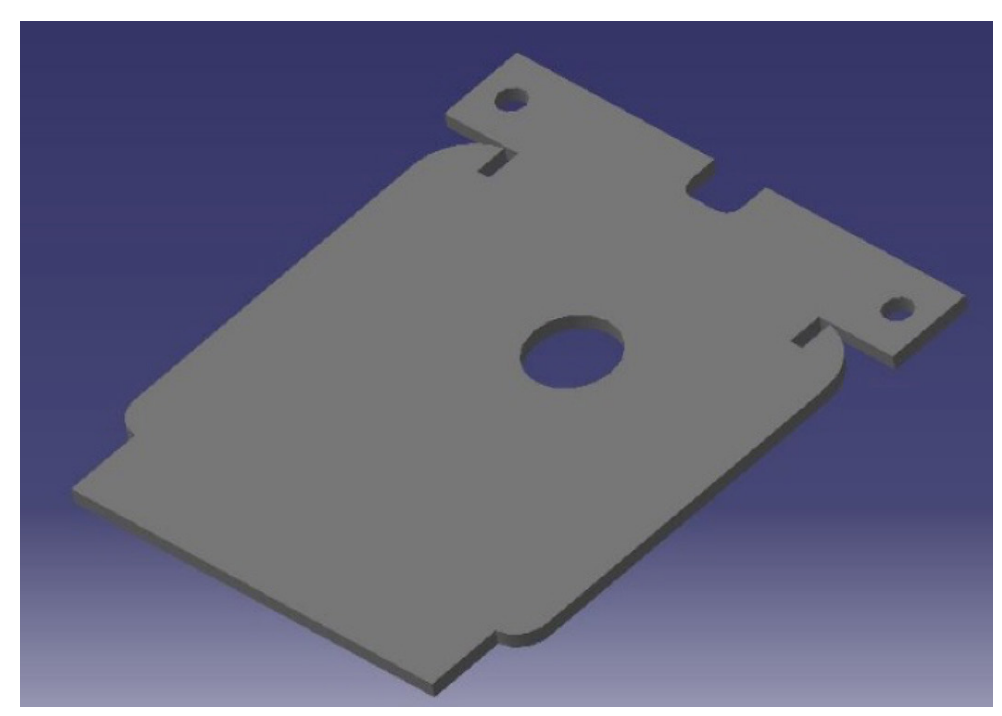

Figura 1. Pieza desplegada. 


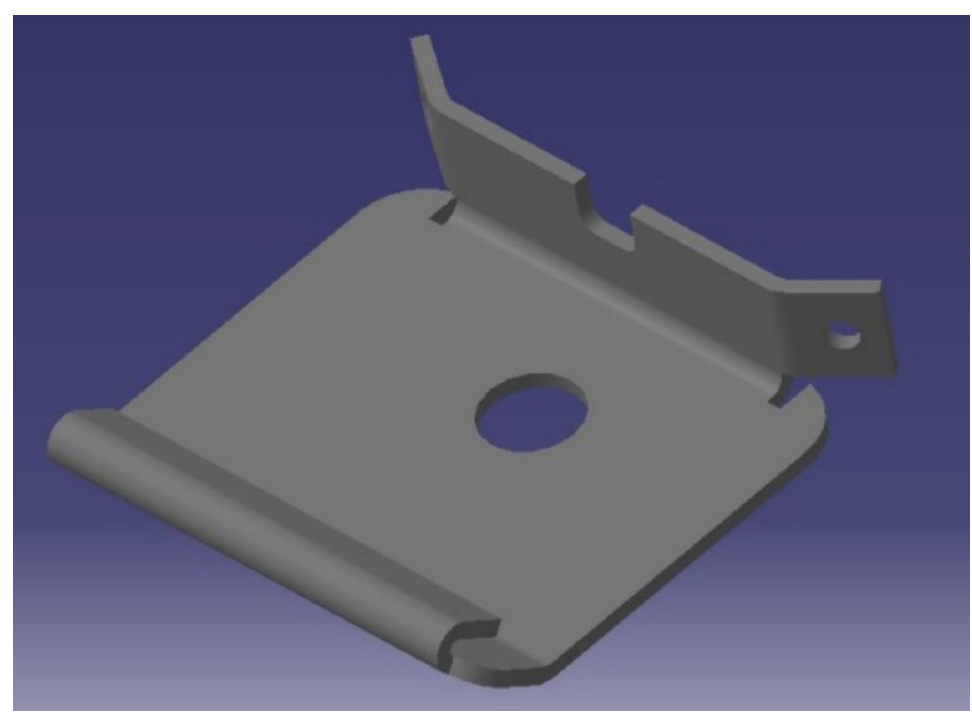

Figura 2. Pieza conformada.

\subsection{Análisis físico del conformado}

El conformado consiste en la deformación de una placa alrededor de un eje recto, teniendo como principales variantes el doblado y el rizado o engargolado. En cada proceso de conformado es imprescindible la ubicación de la fibra neutra con el fin de obtener una pieza con las dimensiones correctas. Para calcular el desarrollo o desplegado de la pieza antes de su doblez, se emplea la Ecuación 1 propuesta por Kalpakjian y Schmid (2006).

$$
L_{b}=\alpha(R+K T)
$$

donde:
Lb es la holgura del doblez
$\alpha \quad$ es el ángulo del doblez
$\mathrm{R} \quad$ es el radio del doblez
$\mathrm{T} \quad$ es el espesor del material
K es la ubicación de la fibra neutra.

En este trabajo se han tomado como muestra los datos de un doblez a $90^{\circ}$, mismo que se realiza a la mitad de la pieza, como se presenta en la figura 3.

A partir de la figura 3 , se realiza la medición de cada uno de los patines (LA) y considerando el espesor T de la pieza y el radio de doblez (R), se emplea la Ecuación 2 propuesta por Kalpakjian y Schmid (2006) para el cálculo del factor K. 
En este caso es necesario que el radio sea igual al espesor del material para facilitar el cálculo; por tanto, $\mathrm{R}=\mathrm{T}$.

La importancia de la Ecuación 2 es la medida que al final se obtiene de los patines, puesto que con esta se puede calcular la ubicación de $\mathrm{K}$, por tanto, no es relevante un valor específico de la longitud inicial de la pieza, la cual se denota por L.
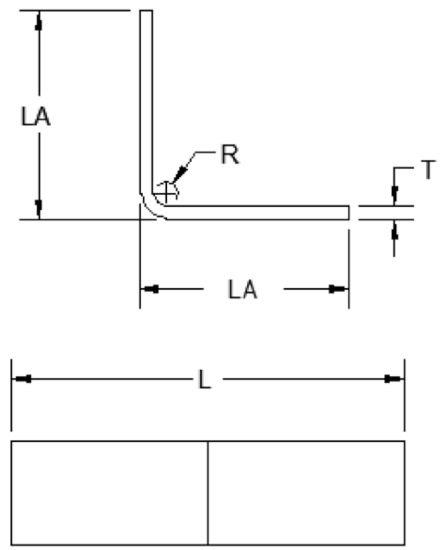

Figura 3.Variables para obtener la fibra neutra. Elaboración propia.

donde:

L representa la longitud del material (paralela al eje de doblez)

LA la longitud del patín

$\mathrm{T} \quad$ el espesor del material

$K=\frac{L-2\left(L_{A}-2 T\right)}{\frac{\pi}{2} T}-1$

La ventaja del uso de la Ecuación 2 es que se toma como base la medida directa de la pieza con un instrumento de medición tal como un vernier o una block y escala, además de no interferir el retroceso elástico generado, puesto que la pieza se puede ajustar hasta lograr el doblez a 90 grados sin que se tenga que realizar algún cálculo fuera de las dimensiones de los patines.

En cualquier proceso de conformado se emplean un punzón y una matriz, como se muestra en la figura 4, los cuales forman el herramental completo. El punzón se desplaza mediante el movimiento rectilíneo de una prensa de conformado y empuja la pieza dentro de la matriz. Su principal importancia es la geometría y basado en ella, el punzón tendrá un radio unitario; es decir, tendrá un radio igual al espesor de la pieza de cada una de las placas de prueba. La matriz tendrá una abertura da de 10 veces el espesor de 
la pieza, normalmente indicado como 10T. La abertura da es la distancia entre apoyos. El empleo de la relación 10T está basado en que, si se aplica una abertura menor, como por ejemplo un $8 \mathrm{~T}$, la pieza contendrá dimensiones menores a las especificadas en el diseño, y por el contrario, si se emplea una abertura mayor la pieza aumenta a dimensiones fuera de las especificaciones de diseño. Lo apropiado es el empleo de relaciones entre $8 \mathrm{~T}$ y $12 \mathrm{~T}$, porque no solo existe afectación en las dimensiones de las piezas, sino que se corre el riesgo de que se fisuren las piezas o el propio herramental.

El material utilizado en este trabajo es el acero inoxidable austenítico. Este acero constituye una familia que integra las series 200 y 300 de la norma AISI (ASM, 1988). Estos aceros tienen una excelente formabilidad y una excelente resistencia a la corrosión (Amor, 2009). Esta familia de aceros es de fácil transformación mecánica, situación por la cual es la preferida para las operaciones de conformado a nivel industrial. Estos aceros presentan la propiedad especial de aumentar su resistencia mecánica en la zona de conformado, conocida como acritud, la que provoca que presenten variaciones en la ubicación de la fibra neutra con el trabajo de conformado en frío. La serie 200 corresponde a las aleaciones cromomanganeso-nitrógeno y la serie 300 corresponde a las aleaciones cromo-níquel (ASTM A480, 2002). En este trabajo se estudiarán específicamente las series 201 y 304, las cuales son empleadas ampliamente en la industria del transporte y principalmente en la ferroviaria. El amplio uso de estos aceros se debe a su alta resistencia ante la corrosión intergranular, así como a su protección ante la corrosión debida a los procesos de soldadura alrededor de las zonas críticas de las juntas y preparaciones para los cordones. La importancia particular del análisis de estos aceros es que en BombardierTransportation más de la mitad de los productos son elaborados con estos materiales y los métodos de ensamble son realizados por medio de soldaduras por arco eléctrico, según reportes de listas de materiales dentro de la empresa. El presente estudio no realiza análisis alguno en cuanto a los métodos de unión, pero la manera de garantizar ensambles correctos se obtiene mediante la elaboración de piezas que actúan como componentes primarios dentro de dimensiones correctas.

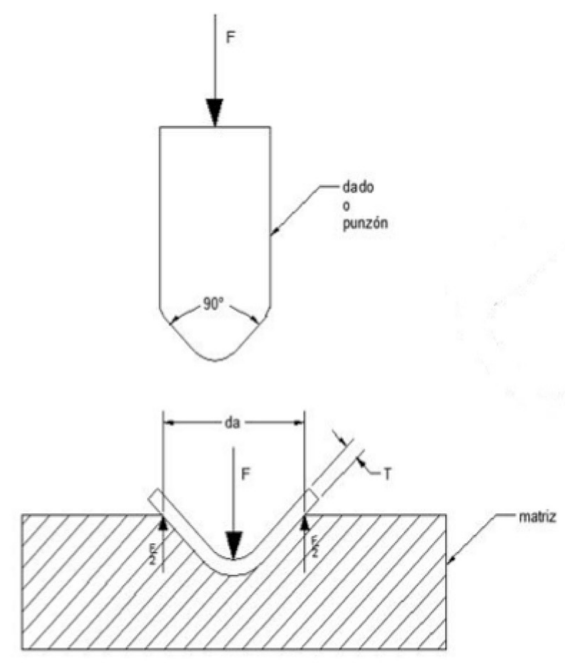

Figura 4. Herramientas de conformado. Elaboración propia. 
Las tablas 1 y 2 muestran, respectivamente, las propiedades mecánicas del acero inoxidable 201 (S20100) y del acero inoxidable 304 (S30400) (Normas ASTM 480M [2002] ASTM A666 [2002] y EN 10088-1 [2015]) que fueron incluidas para el análisis en ANSYS en la sección IV.

Tabla 1. Propiedades mecánicas del Acero Inoxidable 201.

\begin{tabular}{lr}
\hline \multicolumn{1}{c}{ Propiedad } & \multicolumn{1}{c}{ Valor } \\
\hline Densidad & $8,000 \mathrm{Kg} / \mathrm{m}^{3}$ \\
Módulo de Young & $200 \mathrm{e} 9 \mathrm{~Pa}$ \\
Esfuerzo último de tensión & $655 \mathrm{e} 9 \mathrm{~Pa}$ \\
Coeficiente de Poisson & 0.266 \\
Límite Elástico & $310 \mathrm{MPa}$ \\
Dureza Máxima Rockwell B & 95 \\
\hline
\end{tabular}

Tabla 2. Propiedades mecánicas del Acero Inoxidable 304.

\begin{tabular}{lr}
\hline \multicolumn{1}{c}{ Propiedad } & Valor \\
\hline Densidad & $8,000 \mathrm{Kg} / \mathrm{m}^{3}$ \\
Módulo de Young & $200 \mathrm{e} 9 \mathrm{~Pa}$ \\
Esfuerzo último de tensión & $620 \mathrm{e} 9 \mathrm{~Pa}$ \\
Coeficiente de Poisson & 0.3 \\
Límite Elástico & $310 \mathrm{MPa}$ \\
Dureza Máxima Rockwell B & 92 \\
\hline \hline
\end{tabular}

La tabla 3 muestra las fuerzas empleadas en la prensa plegadora para la realización de las pruebas físicas. El doblez empleado fue a $90^{\circ}$. 


\subsection{Análisis por elementos finitos}

Las simulaciones numéricas han permitido el estudio y resolución de diversos problemas de ingeniería (Fuh-Kuo y Shen-Fu, 2006; You-Min, 2003). Actualmente, el uso de simulaciones por computadora, como lo es el análisis por método de elementos finitos (Marciniak, Duncan y Hu., 2002), ha permitido el análisis de estructuras complejas y procesos dinámicos (Al-Momani, 2008). Este tipo de análisis consiste en dividir el cuerpo o estructura en una serie de subdominios denominados elementos finitos, los cuales permiten caracterizar el comportamiento físico de dicho dominio (Amor, 2009). Las ventajas del uso del análisis por elementos finitos son la facilidad de introducir dominios de cálculos complejos, discretizarlos y obtener soluciones tan precisas como los datos de entrada del modelo computacional (Moaveni, 1999).

Tabla 3. Fuerzas aplicadas en la prensa.

\begin{tabular}{|c|c|c|}
\hline Espesor (mm) & Material & Fuerza $(\mathrm{N})$ \\
\hline 3 & AISI 201 & 4,700 \\
\hline 3.17 & AISI 201 & 5,011 \\
\hline 4 & AISI 201 & 6324 \\
\hline 5 & AISI 201 & 7,905 \\
\hline 6 & AISI 201 & 9,485 \\
\hline 3 & AISI 304 & 5,170 \\
\hline 3.17 & AISI 304 & 5,512 \\
\hline 4 & AISI 304 & 6,954 \\
\hline 5 & AISI 304 & 8,695 \\
\hline 6 & AISI 304 & 10,433 \\
\hline
\end{tabular}

Al día de hoy, existe una gran variedad de software que incluye el análisis de elementos finitos, de los cuales una gran mayoría están enfocados a los análisis estructurales y de mecánica de fluidos. En este trabajo se emplea el módulo Explicit Dynamics de ANSYS para modelar el proceso de conformado usando elementos finitos (Moaveni, 1999), pues es un proceso que transcurre de manera muy rápida en el tiempo. El análisis que se ha desarrollado es de tipo plástico, debido a que se analizan las deformaciones del acero inoxidable (Manual TRUMPF, 2013). La caracterización del material se realizó 
usando los datos de las tablas 1 y 2, que presentan las propiedades mecánicas de los aceros empleados. Asimismo, se ha considerado un material de tipo isotrópico y las herramientas se han indicado como elementos rígidos sin fricción.

La figura 5 presenta la simulación realizada en ANSYS, empleando el método de elementos finitos, para el conformado de una pieza. El análisis en ANSYS es del tipo de Von-Mises (Moaveni, 1999), con un tamaño de grano de $0.05 \mathrm{~mm}$ en el modelo de la placa y con un refinamiento de malla de $0.01 \mathrm{~mm}$ en la zona donde se aplica directamente la fuerza del punzón. Las estadísticas del análisis por elementos finitos indican que se utilizaron 92,016 nodos, con un total de 83,070 elementos.

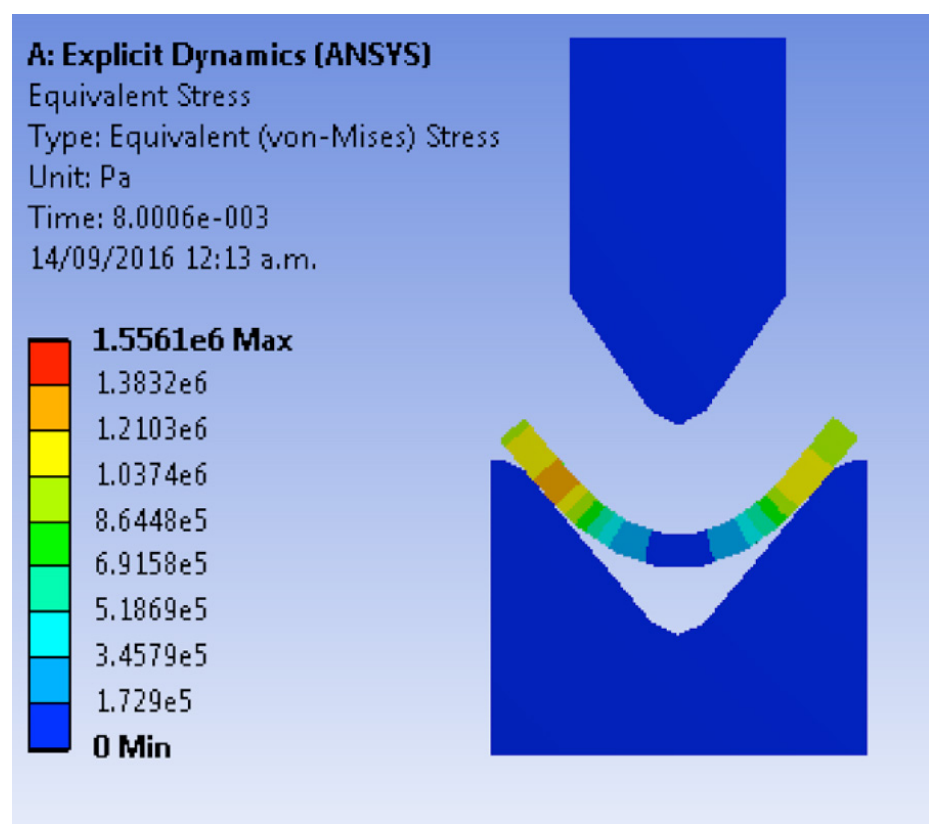

Figura 5. Análisis por elementos finitos en ANSYS.

Se definieron tanto el punzón como la matriz como elementos rígidos, puesto que forma real no existe deformación en ellos, la pieza fue indicada como una conducta de rigidez flexible. La simulación de la bajada de la cortina de la conformadora se situó a una distancia de $6 \mathrm{~mm}$ del material, acorde al proceso real, con una conexión deslizante entre la herramienta y la pieza. Para la solución de la simulación se seleccionó un esfuerzo de tipo Von Mises equivalente y por tiempo. La temperatura de referencia fue indicada como temperatura ambiente. El suavizamiento de la malla fue indicado como alto en los parámetros de mallado.

\section{RESULTADOS}

Los resultados obtenidos mediante las pruebas físicas están tabulados en la tabla 4 y los obtenidos por medio de la simulación en ANSYS se muestran en la tabla 5. 
La figura 6 muestra la comparativa entre las pruebas físicas del acero 201 y las pruebas realizadas por medio de la simulación numérica en ANSYS. En esta figura se observa que los resultados de ANSYS muestran una gráfica parecida en forma a los resultados físicos, aunque el desfasamiento indica que será necesario optimizar aún más el modelo. Sin embargo, los resultados alcanzados en ANSYS son considerados buenos, pues permiten obtener piezas dentro de un rango de tolerancia de menos de $1 \mathrm{~mm}$ en el proceso de conformado, utilizando el factor $\mathrm{K}$ obtenido de manera virtual.

Tabla 4. Ubicación de la fibra neutra (pruebas físicas).

\begin{tabular}{|c|c|c|}
\hline Espesor (mm) & Material & $\begin{array}{c}\text { Ubicación Fibra } \\
\text { Neutra }\end{array}$ \\
\hline 3 & AISI 201 & 0.333 \\
\hline 3.17 & AISI 201 & 0.338 \\
\hline 4 & AISI 201 & 0.339 \\
\hline 5 & AISI 201 & 0.339 \\
\hline 6 & AISI 201 & 0.342 \\
\hline 3 & AISI 304 & 0.334 \\
\hline 3.17 & AISI 304 & 0336 \\
\hline 4 & AISI 304 & 0.337 \\
\hline 5 & AISI 304 & 0.340 \\
\hline 6 & AISI 304 & 0.345 \\
\hline
\end{tabular}

La figura 7 muestra la comparativa entre las pruebas físicas del acero 304 y las pruebas realizadas por medio de la simulación numérica en ANSYS. En esta figura los resultados obtenidos son muy parecidos entre los espesores de 4 a $5.5 \mathrm{~mm}$; no obstante, todavía será necesario optimizar el modelo. Los resultados alcanzados por el método propuesto son buenos, pues para este tipo de acero también permiten obtener piezas dentro de un rango de tolerancia de menos de $1 \mathrm{~mm}$ en el proceso de conformado, utilizando el factor K obtenido de manera virtual.

Las comparativas de la ubicación del factor K entre la simulación en ANSYS y las pruebas físicas de las piezas representó en milímetros un error del 0.4 por ciento. 


\section{CONCLUSIONES}

Diversos estudios han sido realizados sobre el conformado de los distintos aceros inoxidables; sin embargo, están encaminados al estudio de la plasticidad y del retroceso elástico. En este trabajo se analiza una parte muy importante dirigida hacia el corte de la plantilla de doblez con el fin de obtener, al final del proceso de conformado, una pieza dentro de las dimensiones de diseño especificadas. Dicha plantilla se calcula utilizando un factor denominado K. Este factor es la ubicación de la fibra neutra en el espesor del material.

Tabla 5. Ubicación de la fibra neutra (simulación en ANSYS).

\begin{tabular}{ccc}
\hline \hline Espesor $(\mathrm{mm})$ & Material & Ubicación Fibra Neutra \\
\hline 3 & AISI 201 & 0.331 \\
3.17 & AISI 201 & 0.335 \\
4 & AISI 201 & 0.335 \\
5 & AISI 201 & 0.338 \\
6 & AISI 201 & 0.345 \\
3 & AISI 304 & 0.332 \\
4 & AISI 304 & 0333 \\
5 & AISI 304 & 0.337 \\
6 & AISI 304 & 0.340 \\
\hline
\end{tabular}

El método propuesto planteó una simulación virtual explícita con la caracterización de las herramientas y el material a conformar. Mediante la simulación se obtuvo la posición de la fibra neutra para espesores de aceros inoxidables de 3 a $6 \mathrm{~mm}$. Por medio del análisis físico en probetas de aceros inoxidables de la familia 201 y 304, se ha determinado, de manera precisa, la ubicación de la fibra neutra en el proceso de conformado. El error obtenido es de 0.4 por ciento, con base en la comparativa de los resultados de la experimentación física contra los logrados por el modelo virtual. Al ser estos resultados muy aproximados, se determinó que el método propuesto para obtener la ubicación de la fibra neutra es confiable, demostrando su viabilidad para ser empleado en distintos tipos de conformado y utilizando diferentes materiales. Así, usando el método propuesto se busca reducir la cantidad de pruebas físicas desarrolladas en el proceso de conformado, las cuales implican un alto costo en tiempo y materiales invertidos en cada una de ellas. 


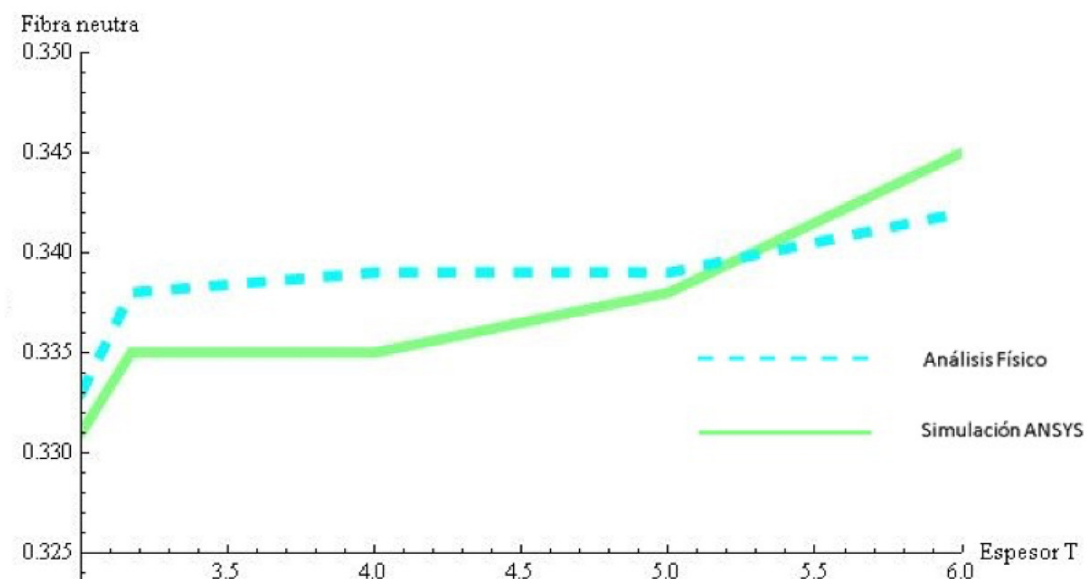

Figura 6. Comparación entre análisis físico y ANSYS para el Acero 201.

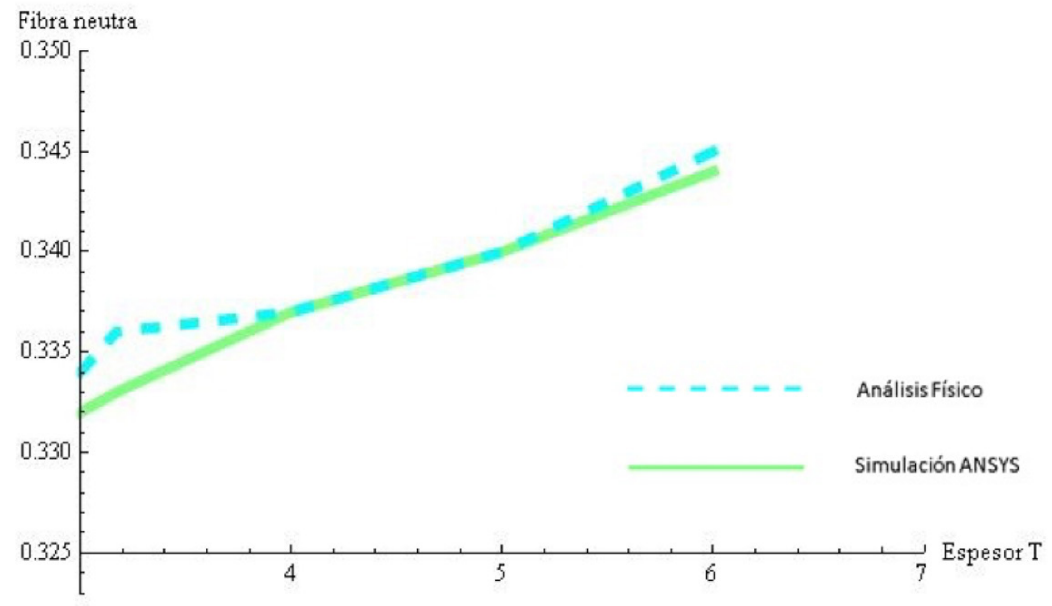

Figura 7. Comparación entre análisis físico y ANSYS para el Acero 304.

El principal problema encontrado en este análisis consiste en que solo puede ser modelado en paquetes computacionales que utilicen análisis explicito, de otra forma los análisis estáticos o dinámicos de programas convencionales no logran proporcionar la geometría final de la piza, sino que únicamente indican los esfuerzos a los que está sometida. Se recomienda que cualquier análisis de conformado para medición directa de la pieza final sea hecha en programas de análisis explícito tales como ANSYS, Midas NFX, MSC Nastran.

Como trabajo futuro se obtendrán y validarán modelos que permitan calcular la fibra neutra en placas de aluminio y materiales estructurales como los tubos redondos y cuadrados de acero estructural e inoxidable. 


\section{REFERENCIAS}

Al-Momani, E. (2008) An Application of Finite Element Method and Design of Experiments in the Optimization of Sheet Metal Blanking Process. Jordan Journal of Mechanical and Industrial Engineering, 2(1), 53-63.

Amor E. (2009). Modelización Numérica del Plegado de los Aceros Inoxidables Austeníticos. Madrid: Escuela Politécnica Superior Carlos III de Madrid.

ASM Handbook Vol.14. (1988). ASM International.

ASTM A480/A480M. (2002). Standard Requirements for Flat-Rolled Stainless and Heat-Resisting Steel Plate, Sheet, and Strip. ASTM International.

ASTM A666/A666M. (2002). Specification for Austenitic Stainless Steel Sheet, Strip, Plate, and Flat Bar. ASTM International.

Balon, P., Swiatoniowski, A. y Szostak, J. (2016). Improved Method of Springback Compensation in Metal Forming Analysis. Journal of Kones Powertrain and Transport, 19(3), 459-468.

Banabic, D. (2010). Sheet Metal Forming Processes. Rumania: Springer.

Castro, L. F. (2010). Condiciones Técnicas para un Correcto Doblado. Metal Actual, 15(5), 16-23

EN 10088-1:2014. (2015). Stainless steels. España: AENOR.

Fuh-Kuo, C. y Shen-Fu, K. (2006). Deformation Analysis of Springback in L-bending of Sheet, Metal. Journal of Achievements in Materials and Manufacturing Engineering, 18(2), 339-342.

García-Romeu, M. L. (2005). Contribución al Estudio del Proceso de Doblado al Aire de Chapa. Modelo de Predicción del Ángulo de Recuperación y del Radio de Doblado Final. Girona: Universidad de Girona.

Kalpakjian, S. y Schmid, S. (2006). Manufacturing Engineering and Technology. Estados Unidos: Editorial Prentice Hall.

Lange, K. (1997). Modern Metal Forming Technology for Industrial Production. Journal of Materials Processing Technology, 71(1), 2-13.

Laufgang, S. (2003). Aceros Inoxidables. Perú: Termo Soldex S.A.

Manual TRUMPF. (2013). Estados Unidos: Bending Technology.

Marciniak, Z., Duncan, J.L. y Hu S.J. (2002). Mechanics of Sheet Metal Forming. Inglaterra: Butterworth Heinemann.

Moaveni, S. (1999). Finite Element Analysis. Theory and Applications with ANSYS. Estados Unidos: Prentice Hall.

Webb, R. D. y Hardt, D. E. (1991). A Transfer Function Description of Sheet Metal Forming for Process Control. Journal of Engineering for Industry, 44(5), 44-52.

You-Min, H. (2003). An Elasto-Plastic Finite-Element Analysis of Sheet Metal Camber Process. Journal of Materials Processing Technology, 140(20), 432-440.

Zahid, F., Waheed, S. y Liaqat, A. (2016). Sheet Metal Bend Sequence Planning Subjected to Process and Material variations. The International Journal of Advanced Manufacturing Technology, 88(4), 815-826. 